\title{
Zur Kenntniss des sogenannten thierischen Gummis.
}

\author{
Von \\ Otto Folin (Chicago).
}

(Der Redaction zugegangen am 20. Mai 1897.)

Diese Arbeit ist auf Anregung des Herrn Professor Hammarsten in seinem Laboratorium ausgeführt worden. Die Hauptaufgabe derselben war das Studium des aus dem Submaxillarismucin beim Sieden mit Säuren entstehenden, seiner Natur nach noch wenig bekannten, reducirenden Spaltungsproduktes; da ich aber in erster Linie eine Nachprüfung der zur Darstellung des sogenannten thierischen Gummis verwendeten Methoden unternehmen musste, will ich in diesem Aufsatze nur über diesen Theil meiner Arbeit berichten. Die wichtigsten Angaben über das thierische Gummi rühren bekanntlich von Landwehr her. ${ }^{1}$ ) Er stellte nämlich aus der Submaxillarisdrüse und dann aus vielen anderen Organen eine angeblich stickstofffreie Substanz dar, die selbst nicht reducirend wirkte, nach dem Sieden mit einer Säure aber eine reducirende Zuckerart geben sollte. Er nannte diese Substanz thierisches Gummi und schrieb ihr die Zusammensetzung $\mathrm{C}_{12} \mathrm{H}_{20} \mathrm{O}_{10}$ zu. Das Gummi stellte er nicht nur aus Mucin, sondern auch aus verwandten Substanzen, wie dem Paralbumin, dem Metalbumin und dem Chondrin dar, und er betrachtet dementsprechend Mucin und Chondrin als die zwei Hauptquellen für die Darstellung dieser Substanz.

1) Diese Zeitschrift 5, 6, 8, 9. Pflügers Archiv 39, 40. Centralblatt f. d. med. Wissenschaft 1885, Nr. 21. 
Die Richtigkeit dieser Angaben ist mehr oder weniger allgemein angenommen worden und man spricht in der Litteratur von dem Vorhandensein dieses thierischen Gummis wie von einer sichergestellten Thatsache, trotzdem die Angaben Landwehrs von den Nachuntersuchern eigentlich nur wenig Bestätigung gefunden haben. Mit Ausnahme von Loebisch scheinen nämlich sämmtliche Forscher, welche auf diesem Gebiete gearbeitet haben, zu andern Resultaten gekommen zu sein.

Loebisch wiederholte die Untersuchungen Landwehrs an einem anderen Materiale, nämlich dem Mucin der Achillessehne, und er erhielt dabei als Endprodukt eine Substanz, die in qualitativer Hinsicht wie das Gummi sich verhielt, und welche für $\mathbf{C}$ und $\mathbf{H}$ Zahlen gab, die zu der Formel eines Kohlenhydrates gut stimmten. ${ }^{1}$ ) Landwehr behauptet wiederholt, dass sein Gummi stickstofffrei sei; aber er spricht nie davon, in welcher Weise er die Substanz auf einen Gehalt an Stickstoff prüfte. Ebensowenig macht Loebisch eine Angabe hierüber, und man kann aus seinem Aufsatze nicht ermitteln, ob er die Substanz direkt auf Stickstoff geprüft hat, oder ob er wegen der Uebereinstimmung in den qualitativen Reactionen wie in dem C- und H-gehalte die Identität mit dem Landwehr'schen Gummi angenommen hat. Dieser Mangel an bestimmten Angaben über die Art und Weise, wie er auf Stickstoff prüfte, ist um so mehr zu bedauern, als die von Loebisch dargestellte Substanz eine Säure war, die Carbonate unter $\mathrm{CO}_{2}$-entwicklung zersetzte.

Die Existenz des Landwehr'schen Gummis scheint auch in der Arbeit Hammarsten's über das Mucin der Weinbergschnecke (Helix Pomatia) gewissermassen eine Bestätigung gefunden zu haben $\left.{ }^{2}\right)$. Hammarsten erhielt nämlich aus dem Mantelmucin dieses Thieres durch Alkalibehandlung ein Mal eine kleine Menge einer Substanz, welche die qualitativen Reactionen des thierischen Gummis gab und die bei der Prüfung mit der Lassaigne'schen Probe als stickstofffrei sich erwies.

1) Diese Zeitschrift, Bd. 10. S. 40.

2) Pflügers Archiv 36, S. 409. 
Dies gelang ihm indessen, wie oben gesagt, nur ein Mal, trotzdem er den Versuch drei Mal wiederholte. Professor Hammarsten hat mich ferner autorisirt, hier mitzutheilen, dass er nunmehr, seitdem er sich wiederholt von der Unzuverlässigkeit der Lassaigne'schen Probe bei kleinen Substanzmengen überzeugt hat, für die Abwesenheit von Stickstoff in der obengenannten kleinen Substanzmenge nicht einstehen kann. Mit Rücksicht auf das Submaxillarismucin hat Hammarsten ${ }^{1}$ ) dagegen ganz bestimmt erklärt, dass er aus demselben nie ein stickstofffreies Kohlenhydrat hat darstellen können. Er erhielt stets sauer reagirende, stickstoff haltige Spaltungsprodukte.

Noch schlimmer steht es aber mit der Angabe Landwehr's über das Vorkommen von thierischem Gummi in dem Knorpel. Schmiedeberg hat nämlich gezeigt, dass die nach Landwehr's Methode aus Chondrin darstellbare Substanz zwar die Reactionen des sogenannten thierischen Gummis gibt, dass sie aber ein Gemenge von Chondroitin und Chondroitinschwefelsäure ist und dementsprechend sowohl $\mathbf{N}$ wie $\mathbf{S}$ enthält ${ }^{2}$ ).

Andere Forscher, wie Müller ${ }^{3}$ ) und Weydemann ${ }^{4}$ ), die in der letzten Zeit, ob zwar nach anderen Methoden, auf diesen Gebiete arbeiteten, haben ebenfalls nur stickstoffhaltige Substanzen erhalten; und das Auftreten eines sogenannten thierischen Gummis als Spaltungsprodukt der Mucinsubstanzen muss also mindestens als sehr zweifelhaft angesehen werden.

Da indessen die Angaben Landwehr's sehr bestimmt lauten, und da es ferner für mich von Wichtigkeit war, gerade das reine Gummi wenn möglich als Ausgangsmaterial für meine Arbeit zu machen, so lag es mir in erster Linie ob, das thierische Gummi genau nach den Angaben von Landwehr ${ }^{5}$ ) darzustellen. burg 1896.

1) Hammarsten's Lehrbuch d. physiol. Chemie, 3. Auflage. S. 39.

2) Archiv f. exp. Path. u. Pharm. 28, S. 377.

3) Gesellschaft d. Gesammt-Naturwissen. zu Marburg 1896, Nr. 6.

4) "Ueber das thierische Gummi." Inaugural-Dissertation, Mar-

5) Diese Zeitschrift, Bd. 8. S. 165. 
Man stösst indessen hierbei sogleich auf die Schwierigkeit, dass Landwehr keine hinreichend detaillirte Angaben geliefert hat. So sagt er zwar, dass er die fein zertheilten Organe im Papin'schen Topfe kocht, gibt aber keine Angaben über die Temperatur an. Aus diesem Grunde musste ich die Substanz bei verschiedenen Temperaturen, wie $110^{\circ}, 125^{\circ}$ und $150^{\circ}$, erhitzen.

Nach beendetem Erhitzen soll man, nach Landwehr, die colirte Flüssigkeit sehr sorgfältig mit ein wenig verdünnter Essigsäure neutralisiren; dies ist aber eine ganz unverständliche Vorschrift. Die Flüssigkeit reagirt nämlich, wenn man das von mir benutzte Material - Speicheldrüsen vom Rinde -- verwendet, ohne Ausnahme sauer. Die Drüsen reagiren zwar, wenn man sie noch warm von dem geschlachteten Thiere nimmt, alkalisch; beim Erhitzen mit Wasser liefern sie aber innerhalb weniger Minuten eine sauer reagirende Flüssigkeit. Diese saure Reaction rührt zum Theil daher, dass beim Sieden zusammengesetzte Nucleoproteide unter Abspaltung von Nucleinsubstanzen, welche sauer reagirende lösliche Verbindungen darstellen, zersetzt werden; zum Theil rührt sie aber auch von sauren Spaltungsprodukten des Mucins her. Das letztere geht daraus hervor, dass eine mit möglichst wenig Alkali bereitete neutrale Lösung von reinem Mucin in Wasser beim Erhitzen im Digestor sich spaltet und dabei eine saure Lösung gibt. Es ist mir unerklärlich, wie dies Landwehr entgangen ist und wie er von einer Neutralisation der (sauren) Flüssigkeit mit Essigsäure reden kann.

Jch hatte also keine Veranlassung, die gekochte Flüssigkeit mit Essigsäure zu versetzen; um indessen sicher zu sein, dass kein coagulables Eiweiss in ihr zurückgeblieben war, habe ich sie durch wiederholtes Kochen unter Zusatz von Spuren von Alkali oder Säure auf Eiweiss geprüft. Das Resultat war immer ein negatives.

Nach dem Zusatz von Essigsäure setzt Landwehr zu der Flüssigkeit eine sehr kleine Menge Eisenchlorid hinzu und erhitzt zum Sieden, um in dieser Weise das Alkaliacetat unschädlich zu machen. Da in meinen Versuchen der oben- 
genannte Essigsäurezusatz ausblieb, war selbstverständlich auch dieses Verfahren ganz überflüssig.

Meine Lösungen waren also ebenso frei von Eiweiss wie diejenigen von Landwehr, und ich konnte folglich zu der nächsten Stufe der Methode übergehen. Diese besteht darin, dass man die neutralisirte Lösung mit dem gleichen Volumen Alkohol von $80 \%$ versetzt, und hier stimmen unsere Erfahrungen vollkommen überein. Ich konnte nämlich das gleiche Volumen $95 \%$ Alkohol und noch mehr zufügen, ohne eine Fällung zu erhalten.

Nach dem Alkoholzusatze schlägt Landwehr das Gummi durch Zusatz von grösseren Mengen Eisenchlorid und Calciumcarbonat als Eisenverbindung (quantitativ) nieder. Bei der Ausführung dieses Theils des Verfahrens fand ich indessen, was offenbar Landwehr entgangen ist, dass das Eisenchlorid allein fast sogleich einen rothgelben, reichlichen Niederschlag erzeugen kann, der durch einen Ueberschuss des Reagenses nicht gelöst wird. Dieser Niederschlag entsteht regelmässig, gleichgültig ob die Lösung ihre ursprüngliche saure Reaction hat, ob sie neutralisirt oder mit Essigsäure bis zu 0,5\% versetzt worden ist. Er tritt auch ohne Zusatz von Alkohol und bei Zusatz von Eisenchlorid allein auf. Der mit Eisenchlorid allein, ohne Zusatz von Calciumcarbonat, ausgefällte Stoff enthält mehr als $13 \%$ Stickstoff, und ich fand es in Folge dessen sehr wichtig, ihn so vollständig wie möglich zu entfernen. Dies gelingt am sichersten, wenn man so viel Eisenchloridlösung zusetzt, dass die Flüssigkeit nicht weniger als $0,5 \% \mathrm{~F}_{2} \mathrm{Cl}_{3}$ enthält. Eine grössere, sogar die doppelte, Menge Eisenchlorid schadet nicht, was in Hinsicht aut das nun folgende Stadium die Fällung durch Zusatz von $\mathrm{CaCO}_{3}$ von Bedeutung ist.

In Bezug auf diesen letzten Theil des Verfahrens stimmen meine Beobachtungen insofern mit denjenigen von Landwehr überein, als ich bei Zusatz von $\mathrm{CaCO}_{3}$ zu der rothgelben, eisenhaltigen Flüssigkeit den von ihm beschriebenen Niederschlag erhielt. Dieser Niederschlag, mit Chlorwasserstoffsäure und Alkohol nach Landwehr wiederholt gereinigt, gab als Endprodukt eine Substanz, die in qualitativer Hinsicht wie das 
Landwehr'sche Gummi sich verhielt, die indessen nicht als Gummi bezeichnet werden kann. Sie gab nämlich mit Kupfersulfat und Alkali eine blauviolette Lösung (eine Reaction, die von Landwehr nicht erwähnt wird) und sie enthielt eine bedeutende Menge Stickstoff, 10-12\%.

Da ich die Angaben Landwehr's in keinem einzigen Versuche bestätigt fand, dürfte es, damit es dem Leser möglich werde, meine Arbeit zu beurtheilen, bezw. nachzuprüfen, nöthig sein, hier einige detaillirte Versuche anzuführen.

Versuch 1. 2 Kilo fein zerriebene Submaxillardrüsen vom Rinde wurden mit 4 Liter Wasser während $21 / 2$ Stunden in dem Autoclaven auf $125^{\circ} \mathrm{C}$ erhitzt. Die filtrirte, sauer reagirende Flüssigkeit enthielt kein in der Hitze coagulables Eiweiss. $750 \mathrm{ccm}$. mit dem gleichen Volumen Alkohol von $80 \%$ versetzt gaben keine Trübung. Nachdem durch vorläufige Prüfung sich herausgestellt hatte, dass die durch $\mathrm{FeCl}_{3}$ fällbare Substanz durch Zusatz von $0,5 \% \mathrm{FeCl}_{3}$ am vollständigsten sich entfernen liess, und ferner, dass die doppelte Menge Ferrichlorid in keiner Weise die Ausfällung störte, während sie zur Gewinnung eines farblosen Filtrats nach Zusatz von $\mathrm{CaCO}_{3}$ erforderlich war, so wurde das obige Gemenge von Filtrat und Alkohol mit $150 \mathrm{ccm}$. Eisenchloridlösung (von $10 \% \mathrm{FeCl}_{3}$ ) versetzt. Es entstand hierbei ein reichlicher Niederschlag, welcher durch Centrifugiren von der Flüssigkeit getrennt wurde.

Die völlig klare, rothgefärbte Flüssigkeit wurde in der Kälte mit $\mathrm{CaCO}_{3}$ versetzt und kräftig geschüttelt. Nach einigen Minuten war die Reaction beendet; ich erhielt einen reichlichen hell rothbraunen Niederschlag und eine vollkommene farblose klare Flüssigkeit. Der Niederschlag wurde wiederholt mit destillirtem Wasser ausgekocht, zwischen Papier etwas gepresst und dann nach Landwehr in möglichst wenig Chlorwasserstoffsäure von $25 \%$ gelöst. Diese Lösung wurde mit Alkohol gefällt und der Niederschlag über Nacht unter Alkohol stehen gelassen.

Der Niederschlag war nun nicht ganz vollständig löslich in heissem Wasser und es wurde deshalb durch Warmwassertrichter heiss filtrirt. Beim Erkalten schied sich wieder ein wenig Substanz aus, die in Folge der Schwerfiltrirbarkeit der ziemlich concentrirten Lösung durch Centrifugiren abgetrennt wurde. Die klare Flüssigkeit wurde nun wiederum mit Alkohol versetzt, konnte aber erst nach Zusatz von ein wenig $\mathrm{NaCl}$ vollständig gefällt werden. Am folgenden Tage wurde der Niederschlag in heissem Wasser gelöst. Beim Erkalten schied sich auch diesmal ein gallertähnlicher Niederschlag ab, der ebenfalls, um eine möglichst reine Substanz zu erhalten, entfernt wurde. Die Lösung gab beim Sieden mit $\mathrm{CuSO}_{4}$ und Alkali keine Reduction, sondern nur einen blauen Niederschlag, der bei fortgesetztem Sieden sich nicht schwärzte (wichtige Reaction 
nach Landwehr). Nach dem Sieden mit einer Säure während einigen Minuten gab sie starke Reduction mit Kupfersulfat und Alkali. Von Alkohol wurde diese Lösung nicht direct, sondern erst nach Zusatz von ein wenig NaCl-Lösung gefällt und nach $\mathrm{L}$ andwehr musste sie also völlig rein sein. Inwieweit dies ein Kriterium der Reinheit sein kann, muss dahin gestellt sein, aber unter allen Umständen schien die Substanz die nach dieser Methode überhaupt erreichbare Reinheit $\mathrm{zu}$ besitzen. Frei von Asche (Eisen) ist sie indessen nicht. Land wehr sagt nichts hiervon und er scheint überhaupt nicht seine Substanz auf einen Gehalt an Asche geprüft zu haben. Mir ist es indessen selbst durch wiederholtes Fällen nie gelungen, nach Landwehr's Methode eine ganz aschefreie (eisenfreie) Substanz zu erhalten. Die mit Alkohol und ein wenig $\mathrm{NaCl}$ zuletzt gefällte, durch Stehen unter Alkohol hart gewordene Substanz wurde fein zerrieben, mit heissem Aether extrahirt, dann erst im Exsiccator und darauf bei $1100 \mathrm{C}$ getrocknet und endlich theils zur Stickstoffbestimmung nach $\mathrm{K} \mathrm{Jeld} \mathrm{a} \mathrm{hl} \mathrm{und} \mathrm{theils} \mathrm{zu} \mathrm{einer} \mathrm{Kohlen-} \mathrm{und} \mathrm{Wasser-}$ stoffbestimmung verwendet.

Die Substanz, welche 2\% Asche (Eisenoxyd) enthielt, hatte folgende Zusammensetzung, auf aschefreie Substanz berechnet:

$$
\mathrm{C}=47,60 \% ; \mathrm{H}=7,20 \% ; \mathrm{N}=9,84 \% \text {. }
$$

In dem obigen Versuche wurde nur $2 \frac{1}{2}$ Stunden auf $125^{\circ}$ C. erhitzt. Wird das Erhitzen bei dieser Temperatur längere Zeit, etwa 4-5 Stunden, fortgesetzt, so erhält man eine sehr dunkle Flüssigkeit, und es ist nicht möglich, ein weisses Endprodukt zu erhalten.

Ich erhitzte deshalb eine andere Portion Drüsenmasse 5 Stunden lang bei $110^{\circ} \mathrm{C}$. und verfuhr darauf ganz wie in dem oben mitgetheilten Versuche. Das als Endprodukt erhaltene Gummi enthielt in diesem Falle 10,33\% N.

$\mathrm{Da}$ ich also weder bei $110^{\circ}$ noch bei $125^{\circ} \mathrm{C}$. ein stickstofffreies Produkt erhielt, versuchte ich das Erhitzen auf $150^{\circ} \mathrm{C}$. Ich erhielt hierbei eine sehr dunkle Flüssigkeit, aus der es unmöglich war, ein weisses Endprodukt darzustellen. Das nach Landwehr's Eisenmethode dargestellte Gummi. war in diesem Falle fast schwarz, enthielt aber 12,4\% N.

In dem Vorigen,(S. 350) habe ich bemerkt, dass ich den von Landwehr vorgeschriebenen Zusatz von sehr wenig Essigsäure zu der von den Drüsenresten getrennten Flüssigkeit 
aus dem Grunde unterlassen habe, weil es erstens nicht möglich ist, eine schon saure Flüssigkeit mit Essigsäure zu neutralisiren, und zweitens, weil ich aus ihr kein coagulables Eiweiss, sei es nach dem Neutralisiren mit Alkali oder nach Zusatz von einer Spur Essigsäure, durch Sieden ausfällen konnte. Dagegen habe ich während meiner Arbeit die Beobachtung gemacht, dass man durch Zusatz von etwas grösseren Mengen Essigsäure schon bei Zimmertemperatur einen Niederschlag erzeugen kann. Den reichlichsten Niederschlag erhält man nach Zusatz von 0,5\% Essigsäure; ein Ueberschuss an Säure löst ihn nicht auf.

Dieser Umstand scheint Landwehr entgangen zu sein, trotzdem er von nicht zu unterschätzender Wichtigkeit ist. Allem Anscheine nach besteht dieser Niederschlag der Hauptsache nach aus einem von der Drüse stammenden Nucleoproteid. Ich schliesse dies theils daraus, dass Hammarsten schon vor 10 Jahren eine solche, das Mucin leicht verunreinigende Substanz in den Drüsen nachgewiesen hat, und theils daraus, dass beim Erhitzen von reinem Mucin mit Wasser bei hinreichend hoher Temperatur keine solche, durch Essigsäure fällbare Substanz entsteht. Wird das Mucin dagegen nur auf $110^{\circ}$ C. erhitzt, so kann man noch eine kleine Portion durch Essigsäure fällbares, verändertes Mucin ausfällen. Ich kann deshalb auch nicht die Möglichkeit in Abrede stellen, dass der Essigsäureniederschlag in einzelnen Fällen aus einem Gemenge von Nucleoproteid mit ein wenig Mucin bestanden hat. Sei dem aber wie ihm wolle, sicher ist es jedenfalls, dass dieser Niederschlag nichts mit dem thierischen Gummi zu thun hat, und aus diesem Grunde musste er entfernt werden.

In der nun folgenden Versuchsreihe entfernte ich also diese Substanz durch Essigsäurezusatz, filtrirte ab, neutralisirte mit Alkali und verfuhr dann wie oben nach Landwehr. Die Resultate waren indessen dieselben wie vorher. Ich erhielt hier ebenso wenig wie in der vorigen Versuchsreihe ein stickstofffreies Gummi; im Gegentheil erhielt ich wiederum eine stickstoffreiche Substanz. Unter den von mir nach diesen Principien ausgeführten Versuchen will ich als Beispiel den folgenden anführen. 
Versuch 2. 1800 gr. fein zerschnittene Drüsenmasse wurden während 4 Stunden mit 3 Liter Wasser auf $110-115^{\circ} \mathrm{C}$. erhitzt: Die Flüssigkeit war bräunlich gefärbt, von saurer Reaction. Sie wurde heiss filtrirt. Die vorläufige Prüfung ergab, dass der reichlichste Essigsäureniederschlag nach Verdünnung mit dem gleichen Volumen Wasser und Zusatz von 0,$50 ; 0$ Essigsäure $z u$ der verdünnten Flüssigkeit zu erhalten war. Nachdem der grauweisse, mit Essigsäure erzeugte Niederschlag zum Boden sich gesetzt hatte, wurde die obenstehende Flüssigkeit abgehebert und einige Tage stehen gelassen. Es trat hierbei kein weiterer Niederschlag auf.

Der obige Niederschlag wurde durch zweimaliges Auflösen in Wasser mit Hülfe von ein wenig Alkali und vorsichtige Ausfällung mit möglichst wenig Salzsäure, gründliches Auswaschen mit Wasser und darauffolgende Alkoholbehandlung.gereinigt. Die Substanz wurde erst im Exsiccator und dann im Luftbade bei $120^{\circ}$ C. getrocknet. Sie enthielt $14,90 \%$ Stickstoff.

Die obige, von dem Essigsäureniederschlage getrennte Flüssigkeit wurde filtrirt, genau neutralisirt und bis auf etwa 1 Liter concentrirt. Infolge der Einwirkung der Essigsäure war sie schon bei Zimmertemperatur etwas dunkler geworden. Bei der Verdunstung nahm die neutralisirte Flüssigkeit wieder eine deutlich saure Reaction an und wurde noch dunkler. Sie wurde von Neuem genau neutralisirt und mit dem gleichen Volumen Alkohol von $95 \%$ versetzt. Es fand hierbei keine Trübung statt.

Es wurde nun so viel $10 \%$ ige Eisenchloridlösung zugesetzt, dass das Gemenge $0,5^{0} ; 0$ davon enthielt. Hierbei trat nur ein verhältnissmässig kleiner Niederschlag auf, der abcentrifugirt wurde. Zusatz von noch mehr Eisenchlorid - dieselbe Menge wie vorher - erzeugte keinen neuen Niederschlag. Nach Zusatz von $\mathrm{Ca} \mathrm{CO}_{3}$ trat sogleich die gewöhnliche, reichliche sogenannte Eisengummifällung auf. Im Laufe der folgenden Nacht vermehrte sich die Fällung nicht unbedeutend. Diese Fällung wurde wiederholt mit Wasser ausgekocht und dann wie gewöhnlich nach $\mathrm{L}$ andwehr mit $\mathrm{HCl}$ und Alkohol gereinigt, bis der Letztere nicht allein, sondern erst nach Zusatz von ein wenig $\mathrm{NaCl}$ einen Niederschlag erzeugte. In der mit Alkohol-Aether gereinigten und dann getrockneten Substanz wurde der Stickstoff, wie gewöhnlich, nach $\mathrm{K} \mathrm{jeld} \mathrm{a} \mathrm{hl} \mathrm{bestimmt.}$ Sie enthielt $13,2 \%$ N.

Nach demselben Principe wurden auch Versuche an-. gestellt mit der nach dem Erhitzen der Drüsen mit Wasser auf $125-128^{\circ} \mathrm{C}$. während 4 Stunden erhaltenen Flüssigkeit. Das Landwehr'sche Endprodukt enthielt in diesem Falle $12,5 \% \mathrm{~N}$. 
Bei den Versuchen mit der Drüsensubstanz ist es mir also in keinem einzigen Versuche gelungen, eine stickstofffreie Substanz zu gewinnen. Im Gegentheil erhielt ich regelmässig stickstoffreiche Substanzen, die zwar in qualitativer Hinsicht wie das Gummi sich verhielten, die aber, ihres hohen Stickstoffgehaltes, $12-13 \%$, wegen den Proteinsubstanzen viel näher standen. Man könnte nun vielleicht geneigt sein, gegen meine Versuche die Einwendung zu machen, dass es mir nicht gelungen war, die Proteinsubstanzen aus der Flüssigkeit oder aus dem Niederschlage zu entfernen. Hierzu kann ich nur antworten, dass ich genau nach den Angaben Landwehr's gearbeitet habe und die einzigen Abweichungen, die ich mir erlaubte, gingen, wie aus dem Vorigen zu ersehen ist, nur darauf hinaus, die Eiweissstoffe noch sicherer zu entfernen. Da meine Flüssigkeiten kein coagulables Eiweiss enthielten, hätte man in ihnen eigentlich nur Albumosen, bezw. Peptone und Leimsubstanzen zu erwarten. Diese Stoffe bleiben aber nach Landwehr alle in der Flüssigkeit zurück, wenn man die Eisenverbindung nach seiner Vorschrift erzeugt und mit Wasser auskocht.

Um indessen die Berechtigung dieser Einwände zu prüfen, war es von Wichtigkeit, auch mit dem ganz reinen Mucin einige Versuche zu machen, und dies habe ich auch gethan.

Die Reindarstellung grösserer Mengen von Submaxillarismucin ist eine recht beschwerliche und zeitraubende Arbeit, die übrigens nur im Winter mit Vortheil auszuführen ist. Allem Anscheine nach wäre ich auch mit meiner $\Lambda$ ufgabe nicht in der vorgesehenen Zeit fertig geworden, wenn nicht Professor Hammarsten eine grosse Menge von trockenem Mucin zu meiner Verfügung gestellt hätte.

Bei meinen Versuchen mit Mucin wurde die Substanz erst in Wasser mit Hilfe von ein wenig 0,04\% Ammoniak in Lösung gebracht. Durch gelindes Erwärmen auf dem Wasserbade und Stehenlassen in der. Kälte über eine Nacht war es hierbei leicht, eine neutrale Lösung darzustellen, die dann in dem Autoclaven auf $110^{\circ}, 125^{\circ}$ oder $150^{\circ} \mathrm{C}$. erhitzt wurde. 
Bei Temperaturen über $120^{\circ}$ C. erhält man regelmässig einen zusammenhängenden festgeronnenen Klumpen, aus dem eiweissartigen Spaltungsprodukte des Mucins bestehend, und eine mehr oder weniger dunkel gefärbte Flüssigkeit, die sauer reagirt, mit Essigsäure keine Fällung gibt und leicht abdekantirt und filtrirt werden kann. (Bei etwa $110^{\circ}$ oder darunter erhält man dagegen kein zusammenhängendes, festes Gerinnsel, sondern einen in der Flüssigkeit ziemlich gleichförmig vertheilten Niederschlag, der abfiltrirt werden muss. Im Laufe von 4 Stunden ist bei $110^{\circ} \mathrm{C}$. nicht alles Mucin gespalten worden, und bei Zusatz von etwas Essigsäure entsteht in dem wasserklaren Filtrate eine Fällung.) Das Filtrat, welches, wenn es nicht zu stark concentrirt wurde, von dem gleichen Volumen Alkohol nicht gefällt wird, behandelte ich in der oben beschriebenen Weise nach Landwehr mit Eisenchlorid und Calciumcarbonat. Die Hauptmasse des Niederschlages entsteht schon nach Zusatz von Eisenchlorid allein, und nur der kleinere Theil erst nach darauffolgendem Zusatz von Calciumcarbonat. Auch die aus reinem Mucin dargestellten, sogenannten Gummipräparate sind reich an Stickstoff. Um dies zu zeigen, führe ich aus dieser Versuchsreihe einen Versuch als Beispiel an.

Versuch 3. $15 \mathrm{gr}$. Mucin wurden in der oben angegebenen Weise in $700 \mathrm{ccm}$. Wasser mit Hilfe von $0,04 \% \mathrm{NH}_{8} \mathrm{zu}$ einer neutralen Flüssigkeit gelöst. Diese Lösung wurde dann während 3 Stunden auf $125^{\circ} \mathrm{C}$. erhitzt, wobei ein festes Coagulum und eine sauer reagirende, völlig klare, aber bräunlich gefärbte Flüssigkeit erhalten wurden. Zusatz von Essigsäure bewirkte keine Trübung und ebenso wenig wurde die Lösung von dem gleichen Volumen Alkohol gefällt. Zu dem Gemenge von Lösung und Alkohol wurde nun Eisenchlorid bis zu $1 \%$ zugesetzt, und es trat hierbei ein reichlicher, rothbrauner Niederschlag auf, der durch Centrifugiren von der Flüssigkeil getrennt wurde. Dieser Niederschlag, mit destillirtem Wasser wiederholt ausgekocht und dann wie oben nach Landwehr gereinigt, enthielt $10,26 \%$ Stickstoff.

Die obige, von dem Niederschlage getrennte eisenreiche Flüssigkeit gab mit $\mathrm{CaCO}_{3}$ einen neuen Niederschlag von dem gewöhnlichen typischen Aussehen. Die Menge desselben war verhältnissmässig klein, und nach dem Auskochen desselben mit Wasser, Zersetzung mit $\mathrm{HCl}$ und Zusatz ron Alkohol wurde nur eine sehr kleine Menge des gummiähnlichen Produktes erhalten. Aus diesem Grunde konnte ich auch nicht 
die Substanz durch wiederholtes Lösen in Wasser und Ausfällung mit Alkohol wie gewöhnlich reinigen, sondern ich musste mich darauf beschränken, sie möglichst gründlich mit Alkohol auszuwaschen. Aus diesem Grunde wurde die Substanz stark von Mineralstoffen, namentlich Eisen, verunreinigt und dies erklärt, warum der Stickstoffgehalt - auf aschenhaltige Substanz berechnet - etwas niedriger als gewöhnlich, $8,73 \% \mathrm{~N}$, war.

Ein dem nun referirten ähnliches Experiment wurde mit 15 gr. desselben Mucins ausgeführt, nur mit dem Unterschiede, dass ich die Flüssigkeit im Topfe auf $150^{\circ} \mathrm{C}$. statt auf $125^{\circ}$ C. erhitzte. Die nun erhaltene Lösung war klar, aber dunkelbraun und sie wurde weder von Essigsäure noch von dem gleichen Volumen Alkohol getrübt. In dieser Flüssigkeit gab Eisenchlorid allein keinen Niederschlag, wogegen eine reichliche Fällung durch Zusatz von $\mathrm{CaCO}_{3}$ erhalten wurde. Das nach Landwehr's Verfahren erhaltene Endprodukt konnte in diesem Falle nicht weiss gewonnen werden. Im Gegentheil war es sehr dunkel gefärbt. Der Gehalt an Stickstoff war 11,4\%.

Es stellte sich also heraus, dass eine Temperatur von $150^{\circ}$ G. für das Mucin ebenso wie für die Drüsen eine nicht geeignete ist, indem bei dieser hohen Temperatur ein Theil der Substanz unter Bildung von schwarzen Produkten zersetzt wird. Ein stickstofffreies Gummi ist bei dieser hohen Temperatur ebensowenig wie bei einer niedrigeren nach dem Landwehr'schen Verfahren zu gewinnen.

Einige qualitative Reactionen, die ich ausgeführt hatte, wie auch der verhältnissmässig hohe Stickstoffgehalt der analysirten Präparate machten es höchst wahrscheinlich, dass bei dem Erhitzen der Drüsen oder einer neutralen Mucinlösung auf die genannten Temperaturen als lösliche Produkte hauptsächlich Mucinalbumosen entstehen, die von dem Eisensalze ausgefällt werden und folglich die Landwehr'sche Eisenmethode für diesen Fall ganz unbrauchbar machen. Aus diesem Grunde war es von Interesse, die Spaltungsprodukte des Mucins beim Erhitzen mit Wasser auch `ohne Anwendung der Eisenmethode $\mathrm{zu}$ isoliren und $\mathrm{zu}$ studiren. Das habe ich auch gethan und als Beispiel führe ich hier den folgenden Versuch an: 
Versuch 4. 15 gr. Mucin, wie oben (S. 357) beschrieben, in $700 \mathrm{ccm}$. Wasser mit Hilfe von $0,04 \% \mathrm{~N} \mathrm{H}_{3} \mathrm{zu}$ einer neutralen Flüssigkeit gelöst, wurden 5 Stunden lang auf $110^{\circ}$ C. erhitzt. Der Niederschlag wurde durch Centrifugiren von der völlig farblosen, sauer reagirenden Flüssigkeit getrennt. Die letztere wurde dann mit Essigsäure bis zu 0,5\% versetzt und der Niederschlag abfiltrirt. Die wasserklare, farblose Flüssigkeit, die nicht weiter mit Essigsäure gefällt werden konnte, wurde mit $\mathrm{KOH}$ genau neutralisirt und auf dem Wasserbade stark concentrirt. Sie nahm hierbei eine schwache bräunliche Färbung an, blieb aber (bis auf einige am Rande der Schale eingetrocknete gummiähnliche Partikelchen) klar. Die klare Flüssigkeit wurde mit dem mehrfachen Volumen Alkohol zersetzt und es schied sich hierbei in reichlicher Menge eine schwach gelblich gefärbte, gummiähnliche Substanz aus, die über Nacht unter Alkohol stand. (Die Ausfällung war eine so vollständige, dass in dem alkoholischen Filtrate nach dessen Verdunstung bei $60^{\circ} \mathrm{C}$. nur Spuren von Substanz zurückgeblieben waren. Dieser Rückstand war zum Theil in Alkohol unlöslich, zum Theil bestand er aber aus einer in Alkohol löslichen, die Fehling'sche Flüssigkeit reducirenden Substanz).

Der obengenannte, mit Alkohol erzeugte gummiähnliche Niederschlag wurde in Wasser gelöst und die filtrirte Lösung mit überschüssigem Alkohol versetzt. Es trat hierbei allerdings ein Niederschlag auf, die Fällung war aber erst nach Zusatz von ein wenig NaCl-Lösung eine vollständige. Dieser Niederschlag, wiederum in Wasser gelöst, gab eine Lösung, die folgendermassen sich verhielt.

Die Heller'sche Probe gab, wie in Mucinalbumoselösungen, einen durchsichtigen bläulich-weissen Ring, der bei Umrühren verschwand. Keine Fällung. Trübung.

Essigsäure allein oder Essigsäure und Ferrocyankalium, keine schlag.

Gerbsäure gab erst in grösserer Menge einen bleibenden Nieder-

Kupfersulfat allein, keine Trübung. Mit Kupfersulfat und Alkali eine rothviolette Lösung. Mit einer grösseren Menge des Kupfersalzes und Alkali wurde ein bläulich-weisser Niederschlag erhalten, der wie der entsprechende Gummikupferniederschlag von Landwehr sich verhielt, indem er nämlich beim Sieden sich nicht schwärzte. Eine sichtbare Reduction fand hierbei nicht statt.

Eis enchlorid erzeugte in der wässerigen Lösung keine Fällung, durch Zusatz von Alkohol zu der gelbgefärbten Lösung trat aber der gewöhnliche rothbraune Niederschlag auf.

Alkohol allein, selbst Zusatz von 7-8 Volumen Alkohol (95\%), machte die Lösung nur bläulich-weiss opalisirend. Nach Zusatz von ein wenig $\mathrm{Na} \mathrm{Cl}$ kam sogleich ein reichlicher, schneeweisser, flockiger Niederschlag zum Vorschein. 
Diese Substanz, die offenbar eine Mucinalbumose war oder jedenfalls reichliche Mengen Mucinalbumose enthielt, verhielt sich also in den wesentlichen Punkten wie das Landwehr'sche Gummi. Sie gab allerdings die Biuretreaction, da aber Landwehr in seiner Arbeit dieser Reaction keine Erwähnung thut, lässt sich aus derselben kein Schluss, sei es für oder gegen die Identität der zwei Substanzen, ziehen. Die untersuchte Substanz enthielt $10 \%$ N. Die Lösung dieser Substanz wurde nun nach Landwehr behandelt. Nach Zusatz von dem gleichen Volumen Alkohol setzte ich Eisenchlorid bis $\mathrm{zu} 1 \%$ und unmittelbar Calciumcarbonat hinzu. Ich erhielt so eine reichliche Eisenfällung und eine farblose Flüssigkeit. Der Niederschlag wurde wie gewöhnlich mit Wasser ausgekocht, nach Landwehr mit Säure und darauf mit Alkohol behandelt und durch wiederholtes Auflösen in Wasser und Fällung mit Alkohol gereinigt, bis die Lösung nicht mehr von Alkohol allein, ohne Salzzusatz, gefällt wurde. Die so gereinigte Substanz enthielt 9,67\% N.

Die mit Alkohol direkt gefällte Mucinalbumose hatte also fast denselben Stickstoffgehalt $(10 \%)$ wie die in der Eisenfällung enthaltene Suhstanz $(9,67 \%$ ). Diese letztere war reicher an Asche, namentlich Eisen, dessen Menge ich indessen wegen Mangels an Substanz nicht bestimmen konnte, und es ist sehr wahrscheinlich, dass, wenn ich in beiden Fällen den Stickstoff auf aschefreie Substanz hätte berechnen können, ich für beide dieselben Zahlen erhalten hätte. Der Unterschied ist aber jedenfalls so klein, dass kein Zweifel darüber bestehen kann, dass die Mucinalbumose von dem Eisensalze gefällt wird.

Die Fällbarkeit der Mucinalbumoseverbindungen mit Eisen hat Hammarsten übrigens schon vor vielen Jahren benutzt, um solche in Verbindungen darzustellen. Ein solches nach einem abweichenden Verfahren dargestelltes Präparat, welches mehr als 6 Jahre alt ist und trotzdem in Wasser vollständig löslich war, habe ich auf den Gehalt an Stickstoff und Eisen untersucht. Ich fand darin $10,4 \% \mathrm{~N}$ und $8 \% \mathrm{Fe}_{2} \mathrm{O}_{3}$.

Die Mucinalbumose kann durch eine hinreichende Menge Gerbsäure ausgefällt werden und ich habe deshalb auch versucht, die nach dem Erhitzen des Mucins im Digestor auf $125^{\circ}$ C. erhaltene Flüssigkeit durch Zusatz von Gerbsäure von der Albumose zu befreien. Nach der vollständigen Ausfällung mit Gerbsäure setzte ich dem Filtrate Alkohol in reichlicher Menge hinzu, konnte aber nur einen winzigen, zur weiteren 
Verarbeitung gar nicht hinreichenden Niederschlag erhalten. Auch in dieser Weise gelang also der Nachweis von Gummi nicht.

Durch die nun mitgetheilten Versuche sind also folgende zwei Thatsachen festgestellt worden: 1. Beim Erhitzen von Speicheldrüsen vom Rinde oder von neutralen Lösungen des entsprechenden Mucins bei $110^{\circ}-150^{\circ} \mathrm{C}$. in dem Autoclaven während mehrerer Stunden erhält man in der Lösung ein verändertes Mucin, welches am passendsten Mucinalbumose genannt wird. Diese Albumose stellt bei Verarbeitung von reinem Mucin (neben Spuren einer reducirenden Substanz und bei höheren Temperaturen braunen oder schwarzen, humusähnlichen Produkten) das in Lösung übergehende Hauptprodukt der Spaltung dar; 2. Die Albumose wird von der Eisenchloridlösung unter den von Landwehr angegebenen Bedingungen gefällt. Die Eisenverbindung löst sich nicht in siedendem Wasser ${ }^{1}$ ), wird aber nach der Zersetzung der Verbindung mit Säure von Alkohol gefällt. Die Eisenmethode von Landwehr ist also bei Gegenwart von Mucinalbumose ganz unbrauchbar.

Wie es Landwehr gelingen konnte, unter den oben angegebenen Versuchsbedingungen ein stickstofffreies Kohlenhydrat aus dem Submaxillarismucin zu gewinnen, ist mir völlig unverständlich. Inwieweit die Methode für andere Organe brauchbar sein kann, lasse ich dahingestellt sein, denn meine Beobachtungen beziehen sich nur auf Mucin aus Speicheldrüsen vom Rinde. Für dieses Mucin steht es aber fest, dass es beim Erhitzen mit Wasser kein gummiähnliches Kohlenhydrat, sondern eine solche Albumose gibt, die zu dem Eisensalze wie das sogenannte thierische Gummi sich verhält.

Inwieweit es nach anderen Methoden möglich ist, ein stickstoffärmeres, bezw. stickstofffreies Produkt zu erhalten, darüber will ich mich in diesem Aufsatze nicht aussprechen, denn diesmal war es nur meine Aufgabe, über die Brauchbarkeit der Landwehr'schen Methode zu berichten. Beiläufig kann ich bemerken, dass ich auch die Behandlung mit stark

1) Unter anderen Versuchsbedingungen ist es möglich, auch lösliche Eisenalbumoseverbindungen darzustellen. 
alkalischen Lösungen ohne Erfolg versucht habe, dass es mir aber gelungen ist, nach der Spaltung des Mucins mit Säuren, mit Phenylhydrazin eine osazonähnliche, krystallisirende Verbindung darzustellen.

Zuletzt bleibt es mir noch übrig, dem Herrr Professor Hammarsten für die vielfache Unterstützung, lie während dieser Arbeit von seiner Seite mir zu Theil geworden ist, meinen ergebensten Dank hier abzustatten.

Upsala, Mai 1897. 